\title{
Mitral valve repair for severe mitral valve regurgitation during left ventricular assist device implantation
}

\author{
Amit Pawale, MD, ${ }^{a}$ Shinobu Itagaki, MD, MSc, ${ }^{a}$ Aditya Parikh, MD, ${ }^{b}$ Sean P. Pinney, MD,
}

David H. Adams, MD, ${ }^{a}$ and Anelechi C. Anyanwu, MD

\section{ABSTRACT}

Background: The management of severe mitral regurgitation (MR) at the time of left ventricular assist device (LVAD) implantation is controversial. We adopted an approach of systematic repair of severe MR at the time of LVAD implantation and report our experience.

Methods: We performed mitral valve repair (MVr) on 78 consecutive patients with severe MR undergoing LVAD implantation at our institution between 2013 and 2017. We compared data on these patients to 28 historical controls with severe MR from the immediate preceding period between 2011 and 2013 where the MR was not treated, using Cox modeling and propensity score methods. Median follow-up time was 17.7 months.

Results: Patients who underwent MVr were younger than those who did not (nonMVr group) (55 vs 63 years; $P=.006$ ), but otherwise had similar preoperative characteristics. The incidence of 30 -day mortality $(2.6 \%$ vs $3.6 \% ; P=.78)$ and other early major adverse events was similar in both groups. At 3 months, no patient in the MVr group had more than mild MR compared with 7 patients $(29 \%)$ in the non-MVr group $(P<.001)$. Cardiac catheterization done 3 to 6 months after surgery showed tendency toward greater reduction from preoperative pulmonary artery systolic pressure in the MVr group compared with the non-MVr group ( $-20 \mathrm{vs}-13 \mathrm{~mm} \mathrm{Hg} ; P=.10$ ). The cumulative incidence of readmission due to congestive heart failure at 2 years was lower in the MVr group than in non-MVr group $(7.1 \%$ vs $19.7 \%$; adjusted hazard ratio, 0.18 ; $95 \%$ confidence interval, $0.04-0.76 ; P=.02)$.

Conclusions: Concurrent MVr at the time of LVAD implantation can be done safely without increase in perioperative adverse events. MVr may be associated with better reduction in severity of MR and may have potential benefit in terms of reduction in readmissions for heart failure. ( $\mathrm{J}$ Thorac Cardiovasc Surg 2019;157:1841-8)

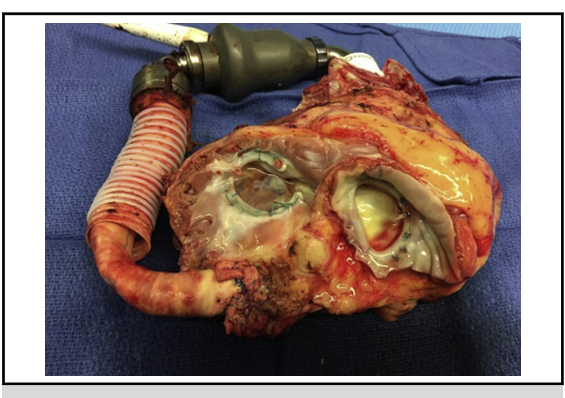

Explanted heart with left ventricular assist device at transplantation with mitral and tricuspid annuloplasties.

\section{Central Message}

Concurrent mitral valve repair at the time of left ventricular assist device implantation can be done safely. It may lead to better reduction in severity of mitral regurgitation and reduced readmissions for heart failure.

\section{Perspective}

Management of severe mitral regurgitation at the time of left ventricular assist device implantation is controversial. This study shows that systematic mitral valve repair can be done safely with minimal incremental risk but further studies are necessary to ascertain the benefit.

See Commentary on page 1849.
A substantial proportion of patients presenting for left ventricular assist device (LVAD) implantation have severe mitral regurgitation (MR). Traditionally, severe MR has not been treated surgically during LVAD implantation. ${ }^{1}$ In the era of pulsatile devices, the unloading of the left

\footnotetext{
From the ${ }^{\mathrm{a}}$ Department of Cardiovascular Surgery and ${ }^{\mathrm{b}}$ Zena and Michael Wiener Cardiovascular Institute, Icahn School of Medicine at Mount Sinai, New York, NY. Read at the 98th Annual Meeting of The American Association for Thoracic Surgery, San Diego, California, April 28-May 1, 2018.

Received for publication May 1, 2018; revisions received Nov 21, 2018; accepted for publication Dec 10, 2018.

Address for reprints: Anelechi C. Anyanwu, MD, Department of Cardiothoracic Surgery, Mount Sinai Medical Center, 1190 Fifth Ave, New York, NY (E-mail: anelechi.anyanwu@mountsinai.org). $0022-5223 / \$ 36.00$

Copyright (C) 2018 Published by Elsevier Inc. on behalf of The American Association for Thoracic Surgery

https://doi.org/10.1016/j.jtcvs.2018.12.071
}

ventricle was such that there was little residual volume in systole, and hence little volume to regurgitate, whereas with the continuous flow devices, significant regurgitation may indicate inadequate left ventricular emptying that could theoretically be overcome by increasing LVAD speed. ${ }^{1}$ Additionally, performing surgery on the left sided heart valves during LVAD implantation had historically

Uf Scanning this QR code will
take you to the article title
page to access supplementary
information. To view the
AATS Annual Meeting Web-
cast, see the URL next to the
webcast thumbnail.




\section{Abbreviations and Acronyms \\ LVAD $=$ left ventricular assist device \\ MR = mitral regurgitation \\ $\mathrm{MVr}=$ mitral valve repair}

been associated with early mortality and morbidity. ${ }^{2}$ Consequently, majority of patients with severe MR having LVAD implantation did not undergo mitral valve surgery. In the era of continuous flow LVADs, anecdotal experience suggests that persisting severe MR after LVAD implantation can complicate patient management due to occurrence of right sided-heart failure, exercise-induced dyspnea, and persistently elevated pulmonary artery pressures. ${ }^{3,4}$ The question arises as to whether MR should be corrected at time of LVAD implantation. We adopted a protocol of systematic repair of severe MR at the time of LVAD implantation in 2013 and report our outcomes.

\section{METHODS \\ Study Design and Population}

Between March 2011 and July 2017, 234 patients underwent LVAD implantation at our institute. There were 209 HeartMate II (Abbott [St Jude Thoratec Corp], Pleasanton, Calif), 14 HeartMate 3 (Abbott [St Jude Thoratec Corp]), and 11 HVAD (HeartWare International, Inc, Framingham, Mass) devices used. All patients presenting for LVAD implantation were systematically evaluated for severe MR using established echocardiographic criteria. ${ }^{5}$ One hundred seven patients $(46 \%)$ with severe MR on either preoperative transthoracic echocardiography or intraoperative transesophageal echocardiography were defined as having severe regurgitation. From April 2013, all patients with severe MR (78 patients) were planned for concurrent mitral valve repair (MVr) at the time of LVAD implantation (the MVr group). Short-term and midterm outcomes were compared with 28 consecutive historical controls (the non-MVr group) from immediately preceding period (March 2011-March 2013), where severe MR was not treated at the time of LVAD implantation. Median follow-up time was 18.9 months in the MVr group and 16.1 months in the non-MVr group, respectively (patients were censored at time of heart transplantation). This study was approved by the Program for Protection of Human Subjects at the Icahn School of Medicine, Mount Sinai Medical Center. The approval included a waiver of informed consent.

\section{Surgical Techniques}

Predominantly, 2 techniques were used: ring annuloplasty or edge-toedge repair.

MVr with ring annuloplasty technique (Video 1). Our technique is described in detail elsewhere. ${ }^{6}$ In summary, the procedure was undertaken via median sternotomy. The pericardium was opened and a pocket was prepared for the ventricular assist device as needed depending on the type of implantable LVAD. After heparinization, the aorta was cannulated (or occasionally a peripheral artery in complex reoperations), as were the superior and inferior vena cavae. Cardiopulmonary bypass with mild hypothermia was instituted. The procedure was typically done without crossclamping and without cardioplegic arrest. If there was another indication for aortic clamping, then cardioplegic arrest was used. The apex of the heart was exposed, the ventricle cored, and the apical sewing cuff attached in a standard fashion. The ventriculotomy was left open to prevent systemic ejection of air during the mitral valve

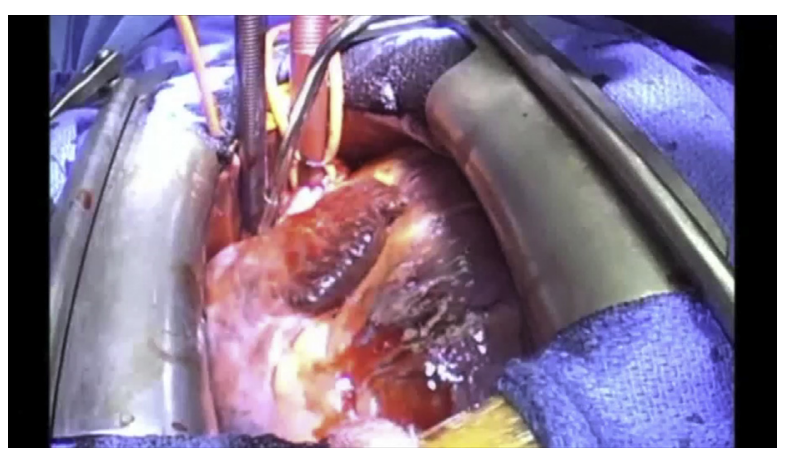

VIDEO 1. Trans-septal mitral valve repair with left ventricular assist device placement. Video available at: https://www.jtcvs.org/article/S00225223(18)33515-3/fulltext.

repair. We usually intentionally induced ventricular fibrillation at time of coring the apex and left the heart fibrillating until the LVAD support was initiated. The mitral valve was accessed via the trans-septal route with care taken to keep the septal incision entirely within the right atrium (sometime this could inadvertently tear and extend to the dome of the left atrium, requiring later repair of the left atrium). A true-sized complete semirigid ring was then implanted using standard techniques. The interatrial septum was closed. A ring annuloplasty was typically also performed on the tricuspid valve after which the right atriotomy was closed. If the outflow graft anastomosis had not been constructed, then this was done with the aid of a side clamp on the ascending aorta. The heart was de-aired through the apex and the inflow cannula was placed and secured. The outflow graft was connected to the pump, the heart defibrillated, and LVAD support was commenced after satisfactory deairing was achieved.

Edge-to-edge repair (Video 2). This was performed transapically and was undertaken either via sternotomy, subcostal, or left thoracotomy approach, depending on the approach used for LVAD implantation. Procedures were performed on cardiopulmonary bypass without aortic crossclamp. After coring the left ventricular apex, the edges of the ventriculotomy were retracted. Cardiotomy suction device was placed in the left atrium through the ventriculotomy. A 3-0 polyester suture with a felt pledget was passed through the mid-section of the posterior, and anterior leaflets in a horizontal mattress fashion few millimeters from the free margin. The suture was secured with fastening device (Cor-Knot; LSI Solutions, Rochester, NY).

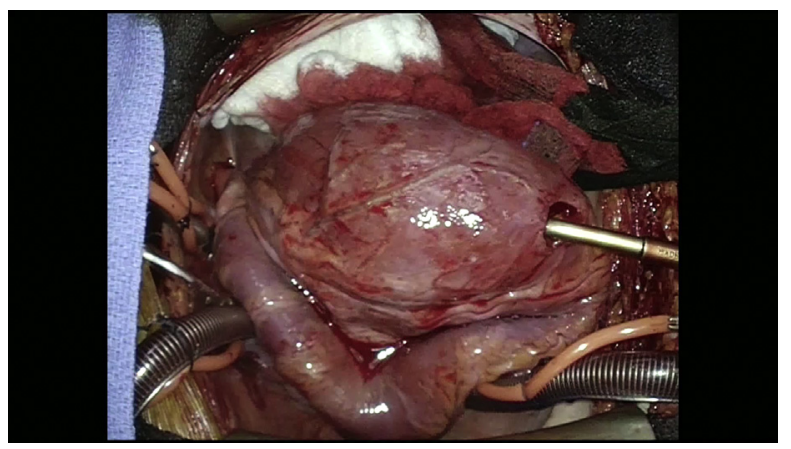

VIDEO 2. Transapical edge-to-edge mitral valve repair with left ventricular assist device placement. Video available at: https://www.jtcvs.org/ article/S0022-5223(18)33515-3/fulltext. 
The choice of repair technique was based on surgeon preference, but there was a bias towards using the edge-to-edge technique if there was a consideration for nonsternotomy LVAD or in sicker patients where an expeditious operation was desirable.

During the study period, 79 patients with severe MR underwent LVAD implantation. One did not undergo MVr and was excluded from further analysis (this was a critically ill patient with multiorgan dysfunction who was being supported with extracorporeal membrane oxygenation support). The 78 remaining patients underwent $\mathrm{MVr}$. Sixty-two patients underwent the ring annuloplasty technique described above. Two patients out of 62 also had leaflet prolapse and required leaflet repair in addition to ring annuloplasty. One with anterior mitral leaflet prolapse received a Gore-Tex (Gore Medical, Flagstaff, Ariz) neochord to A2 and posteromedial commissuroplasty. The other patient had plication of P3. Sixteen patients underwent transapical $\mathrm{MVr}$ without annuloplasty (edge-to-edge leaflet or papillary muscle approximation) via the left ventricular apex after coring. The reasons for the transapical approach were mainly use of less invasive nonsternotomy approach (11 patients), presence of critical cardiogenic shock with multiorgan dysfunction ( 2 patients), and other factors (3 patients). No patient received mitral valve replacement.

\section{Statistical Analysis}

Continuous variables were expressed as means \pm standard deviation if normally distributed or median and range for skewed distributions. Categorical variables were expressed as frequencies and proportions. Differences between groups were evaluated using the $\chi^{2}$ test or Fisher exact test for categorical variables as appropriate and independentsamples Student $t$ test or the Mann-Whitney $U$ test for continuous variables depending on the distribution. Survival curves of the primary end point of all-cause mortality were constructed with Kaplan-Meier methodology and compared with log-rank test. Patients were censored at the time of heart transplantation, LVAD explantation, or last follow-up if neither transplantation nor explantation had occurred. Cumulative incidence curves for the secondary end points of readmission due to heart failure were constructed with competing risk analysis as death and heart transplantation as a competing event and compared with the Gray test. ${ }^{7}$ The multivariable proportional subdistribution hazards model was fit to evaluate the adjusted risk of the secondary end points controlling for concomitant mitral valve repair, age, sex, preoperative systolic pulmonary artery pressure, right ventricular function, tricuspid valve regurgitation, and concomitant tricuspid repair only due to limited number of events and relatively similar baseline comorbidities between the groups. To reduce the potential for overfitting, sensitivity analyses were performed fitting with another model with even more limited number of covariates with concomitant $\mathrm{MVr}$, age, right ventricular function, and tricuspid repair and also with the propensity score adjusted model with only concomitant MVr and propensity scores incorporated as covariates. $^{8}$ Propensity scores were calculated using logistic regression for $\mathrm{MVr}$ adjusting for all preoperative measured covariates (the C-statistic, 0.83). In each model, proportional hazard assumption was evaluated and valid. All statistical analyses were performed using SAS version 9.4 (SAS Institute, Cary, NC).

\section{RESULTS \\ Patients}

The MVr group patients were younger than the non-MVr group patients (55 vs 63 years; $P=.006$ ), but otherwise had similar preoperative demographics and hemodynamics (Table 1). Notably, both groups had significant pulmonary hypertension (56 vs $57 \mathrm{~mm} \mathrm{Hg} ; P=.78$ ) with high pulmonary vascular resistance (4.1 vs $3.5 \mathrm{U} ; P=.26$ ).

\section{Procedure}

In the MVr group, cardioplegic arrest was used in 7 patients for aortic valve repair $(n=4)$, to facilitate outflow graft anastomosis in presence of aortic calcifications $(n=2)$, and for concomitant coronary artery bypass grafting $(n=1)$. The median ring size for MVr group patients was 28. Tricuspid valve repair was performed more frequently in the $\mathrm{MVr}$ group than in the non-MVr group $(\mathrm{n}=68$ out of 78 $[87.2 \%]$ vs $\mathrm{n}=19$ out of $28[67.9 \%] ; P=.02)$. The mean cardiopulmonary bypass time was longer with MVr (142 vs 97 minutes; $P<.001)$. Operative details are found in Table 1.

\section{Immediate Outcomes}

Short-term operative outcomes are found in Table 2. There was no significant difference in re-exploration for bleeding $(\mathrm{n}=5$ out of $78[6.4 \%]$ vs $\mathrm{n}=2$ out of $28[7.1 \%]$; $P=.33)$ or rates number of packed red blood cell transfusion $(\mathrm{n}=28$ out of $78[36 \%]$ vs $\mathrm{n}=14$ out of $28[50 \%] ; P=.19)$ in the repair group compared with no-repair, respectively. Notably, in the MVr group 5 patients underwent reexploration for bleeding - in 3 there was no surgical source found, 1 patient had a chest wall source, and the other had a bleeding epicardial vessel-no bleeding was thought to be directly related to the mitral valve procedure. The incidence of early major adverse events was similar between the groups, including 30-day mortality ( $\mathrm{n}=2$ out of 78 [2.6\%] vs $\mathrm{n}=1$ out of 28 [3.6\%]; $P=.44)$ and stroke ( $\mathrm{n}=2$ out of 78 [2.6\%] vs $\mathrm{n}=1$ out of 28 [3.6\%]; $P=.44$ ). No right ventricular assist device was used in any of the patients in either the intraoperative or postoperative period. Four (5\%) in the MVr group had postoperative right ventricular dysfunction requiring more than 14 days of inotropes compared with $6(21 \%)$ in the non-MVr group. In the MVr group, 1 patient had a pump exchange 2 days after surgery due to device malfunction. Median intensive care unit and hospital stay in the $\mathrm{MVr}$ and non-MVr groups were 4 versus 5 days $(P=.28)$ and 17 versus 22 days $(P=.02)$, respectively. Predischarge transthoracic echocardiography confirmed absence of greater than mild MR in all MVr group patients. Median pump speed at discharge for those who underwent HeartMate II LVAD implantation $(90 \%$ of the study cohort) was $9200 \mathrm{rpm}$ in both groups $(P=.42)$.

\section{Midterm Outcomes and Hemodynamic Parameters Change}

Echocardiography done at median of 3 months postoperatively showed no more than mild MR in any patient in the MVr group ( $n=0$ out of 53) compared with 7 out of 24 $(29.2 \%)$ patients in the non-MVr group ( 2 moderate MR and 5 mild-to-moderate MR). There was no documented occurrence of mitral valve endocarditis, ring dehiscence, or return of more than mild mitral valve regurgitation in the MVr group. More detailed echocardiographic data are 
TABLE 1. Patient demographic characteristics

\begin{tabular}{|c|c|c|c|}
\hline Variable & LVAD with mitral repair $(n=78)$ & LVAD without mitral repair $(n=28)$ & $P$ value \\
\hline \multicolumn{4}{|l|}{ Patient demographic characteristic and comorbidity } \\
\hline Age (y) & $55.1 \pm 13.9$ & $63.1 \pm 9.7$ & .006 \\
\hline Male gender & $64(82.1)$ & $24(85.7)$ & .66 \\
\hline Body mass index & $26.2 \pm 6.5$ & $26.4 \pm 5.2$ & .85 \\
\hline Hypertension & $48(61.5)$ & $22(78.6)$ & .10 \\
\hline Diabetes & $21(26.9)$ & $10(35.7)$ & .38 \\
\hline Cerebrovascular accident & $9(11.5)$ & $4(14.3)$ & .74 \\
\hline Chronic obstructive pulmonary disease & $10(12.8)$ & $1(3.6)$ & .17 \\
\hline \multicolumn{4}{|l|}{ Preoperative status } \\
\hline \multicolumn{4}{|l|}{ Etiology } \\
\hline Ischemic cardiomyopathy & $23(29.5)$ & $8(28.6)$ & .93 \\
\hline Nonischemic cardiomyopathy & $55(70.5)$ & $20(71.4)$ & \\
\hline INTERMACS classification & & & .076 \\
\hline 1 & $6(7.7)$ & $6(21.4)$ & \\
\hline 2 & $17(21.8)$ & $10(35.7)$ & \\
\hline 3 & $48(61.5)$ & $10(32.1)$ & \\
\hline 4 & $4(5.1)$ & $2(7.1)$ & \\
\hline 5 & $3(3.9)$ & $1(3.6)$ & \\
\hline Preoperative mechanical ventilation & $3(3.9)$ & $5(17.9)$ & .025 \\
\hline Preoperative inotropic support & $72(92.3)$ & $24(85.7)$ & .31 \\
\hline \multicolumn{4}{|l|}{ Preoperative hemodynamic characteristic } \\
\hline Ejection fraction $(\%)$ & $17.5 \pm 5.6$ & $18.2 \pm 6.2$ & .62 \\
\hline $\mathrm{LV}$ end-diastolic diameter $(\mathrm{mm} \mathrm{Hg})$ & $71.6 \pm 9.6$ & $70.8 \pm 11.1$ & .70 \\
\hline PA systolic pressure $(\mathrm{mm} \mathrm{Hg})$ & $56.2 \pm 11.4$ & $57.2 \pm 16.1$ & .78 \\
\hline PA mean pressure $(\mathrm{mm} \mathrm{Hg})$ & $38.1 \pm 8.0$ & $38.4 \pm 9.9$ & .86 \\
\hline Pulmonary capillary wedge pressure $(\mathrm{mm} \mathrm{Hg})$ & $26.3 \pm 6.5$ & $28.1 \pm 9.1$ & .29 \\
\hline Central venous pressure $(\mathrm{mm} \mathrm{Hg})$ & $13.3 \pm 6.2$ & $17.1 \pm 6.9$ & .018 \\
\hline Pulmonary vascular resistance $(\mathrm{U})$ & $4.1 \pm 2.9$ & $3.5 \pm 1.8$ & .26 \\
\hline Moderate or greater tricuspid regurgitation & $48(61.5)$ & $13(46.4)$ & .17 \\
\hline Moderate or greater right ventricular dysfunction & $61(78.2)$ & $21(75.0)$ & .73 \\
\hline \multicolumn{4}{|l|}{ Preoperative laboratory data } \\
\hline Hematocrit $(\%)$ & $34(32-38)$ & $30(28-35)$ & .011 \\
\hline Platelet $\left(10^{3}\right)$ & $178(143-234)$ & $166(117-227)$ & .40 \\
\hline Bilirubin (mg/dL) & $1.1(0.7-1.9)$ & $1.1(0.8-1.7)$ & .25 \\
\hline Creatinine (mg/dL) & $1.5(1.2-1.9)$ & $1.7(1.3-2.0)$ & .62 \\
\hline \multicolumn{4}{|l|}{ Surgical details } \\
\hline Destination therapy & $27(34.6)$ & $6(21.4)$ & .38 \\
\hline Tricuspid repair & $68(87.2)$ & $19(67.9)$ & .022 \\
\hline Cardiopulmonary bypass time (min) & $142 \pm 34$ & $97 \pm 21$ & $<.0001$ \\
\hline
\end{tabular}

Values are presented as $\mathrm{n}(\%)$, mean \pm standard deviation, or median (interquartile range). $L V A D$, Left ventricular assist device; INTERMACS, Interagency Registry for Mechanically Assisted Circulatory Support; $L V$, left ventricle; $P A$, pulmonary artery.

provided in Table E1. Cardiac catheterization done within 3 to 6 months of surgery showed a tendency toward greater reduction of the preoperative pulmonary artery systolic pressure in the $\mathrm{MVr}$ compared with non-MVr group (Table 3). The survival at 2 years was $90.2 \%$ (95\% confidence interval [CI], 78.3\%-95.7\%) in the $\mathrm{MVr}$ group and $79.7 \%(95 \% \mathrm{CI}, 57.2 \%-91.2 \%)$ in the non-MVr group (adjusted hazard ratio, $0.47 ; P=.25$ ) (Figure 1). The cumulative incidence of readmission due to congestive heart failure at 2 years was lower in the MVr group at $7.1 \%(95 \% \mathrm{CI}$,
TABLE 2. Operative outcomes

\begin{tabular}{lccc}
\hline \multicolumn{1}{c}{ Variable } & $\begin{array}{c}\text { LVAD with } \\
\text { mitral repair } \\
(\mathbf{n}=\mathbf{7 8})\end{array}$ & $\begin{array}{c}\text { LVAD without } \\
\text { mitral repair } \\
(\mathbf{n}=\mathbf{2 8})\end{array}$ & $\boldsymbol{P}$ value \\
\hline $\begin{array}{l}\text { Packed red blood cell } \\
\text { transfusion }\end{array}$ & $28(35.9)$ & $14(50.0)$ & .19 \\
Re-exploration for bleeding & $5(6.4)$ & $2(7.1)$ & .32 \\
Stroke & $2(2.6)$ & $1(3.6)$ & .43 \\
30-d Mortality & $2(2.6)$ & $1(3.6)$ & .43 \\
\hline
\end{tabular}

Values are presented as $\mathrm{n}(\%)$. LVAD, Left ventricular assist device. 
TABLE 3. Hemodynamic parameters 3 months after left ventricular assist device (LVAD) implantation

\begin{tabular}{|c|c|c|c|c|c|}
\hline Variable & $\begin{array}{l}\text { LVAD with } \\
\text { mitral repair }\end{array}$ & $\begin{array}{l}\text { Change from } \\
\text { pre-operation }\end{array}$ & $\begin{array}{l}\text { LVAD without } \\
\text { mitral repair }\end{array}$ & $\begin{array}{l}\text { Change from } \\
\text { pre-operation }\end{array}$ & $P$ value \\
\hline LVEDD (mm) & $58.3 \pm 13.6$ & $-15.3 \pm 11.6$ & $57.5 \pm 12.6$ & $-13.5 \pm 11.4$ & .53 \\
\hline PA systolic (mm Hg) & $36.5 \pm 8.9$ & $-19.6 \pm 15.4$ & $43.0 \pm 13.3$ & $-12.5 \pm 19.0$ & .10 \\
\hline PA mean $(\mathrm{mm} \mathrm{Hg})$ & $24.1 \pm 6.3$ & $-13.6 \pm 11.1$ & $28.0 \pm 9.2$ & $-10.1 \pm 12.3$ & .24 \\
\hline PCWP (mm Hg) & $13.7 \pm 6.4$ & $-12.1 \pm 8.7$ & $16.4 \pm 6.9$ & $-11.5 \pm 11.0$ & .85 \\
\hline CVP (mm Hg) & $9.0 \pm 5.1$ & $-3.3 \pm 6.5$ & $10.7 \pm 7.0$ & $-5.3 \pm 8.6$ & .28 \\
\hline PVR (U) & $2.4 \pm 1.0$ & $-1.7 \pm 3.1$ & $2.6 \pm 0.9$ & $-0.79 \pm 2.6$ & .27 \\
\hline
\end{tabular}

Values are presented as mean \pm standard deviation. Data are derived from right heart catheterization data obtained at median follow-up time of 3.9 months in the mitral group ( $\mathrm{n}=$ 55 out of 78$)$ and 3.7 months in the nonmitral group $(\mathrm{n}=20$ out of 28$)$. LVAD, Left ventricular assist device; $L V E D D$, left ventricular end-diastolic diameter; $P A$, pulmonary artery; $P C W P$, pulmonary capillary wedge pressure; $C V P$, central venous pressure; $P V R$, pulmonary vascular resistance.

$6.9 \%-37.3 \%$ ) than the non-MVr group at $19.7 \%(95 \% \mathrm{CI}$, $2.2 \%-16.0 \%$ ) (adjusted hazard ratio, $0.18 ; 95 \%$ CI, 0.04$0.76 ; P=.02$ ) (Figure 2 ). The sensitivity analyses validated these findings (Table E2). The transplant surgeons did not experience any specific difficulties at transplantation that were attributed to the MVr.

\section{DISCUSSION}

Our data demonstrate that it is feasible to routinely repair the mitral valve at time of implantation of continuous flow LVAD. The procedure can be applied to a wide variety of patients and scenarios and can be done safely, with acceptable early and late outcomes. We were effective in eliminating severe MR in all MVr patients, and this persisted through the follow-up period. We also demonstrated propensity to reduction in pulmonary artery pressures and reduced rate of readmissions with congestive heart failure.

There are theoretical reasons to support elimination of mitral regurgitation by repair of the mitral valve at time of LVAD implantation. Although the volume of MR will typically decrease following implantation of $\operatorname{LVAD}^{9}$; the effectiveness of continuous flow LVADs in unloading the left ventricle, and therefore reducing MR, is variable. In theory, higher revolutions per minute LVAD speeds should abolish regurgitation; however, this is not consistent, and some patients remain with clinically significant regurgitation, despite optimal revolutions per minute settings. Furthermore increasing the revolutions per minute to offset MR can result in worsening right ventricular function due to septal shift. ${ }^{10}$ Sometimes severe functional MR cannot be eliminated by increase in revolutions per minute, ${ }^{3}$ suggesting that some patients may have a geometric substrate for MR that is independent of ventricular volume. In one study of 90 LVAD recipients, $44 \%$ of patients were observed to have at least moderate MR in the follow-up period. ${ }^{11}$ Another study found that $34 \%$ of patients remained with significant late $\mathrm{MR}^{12}$; severe tethering of the valve was associated with persistence of MR leading the authors to

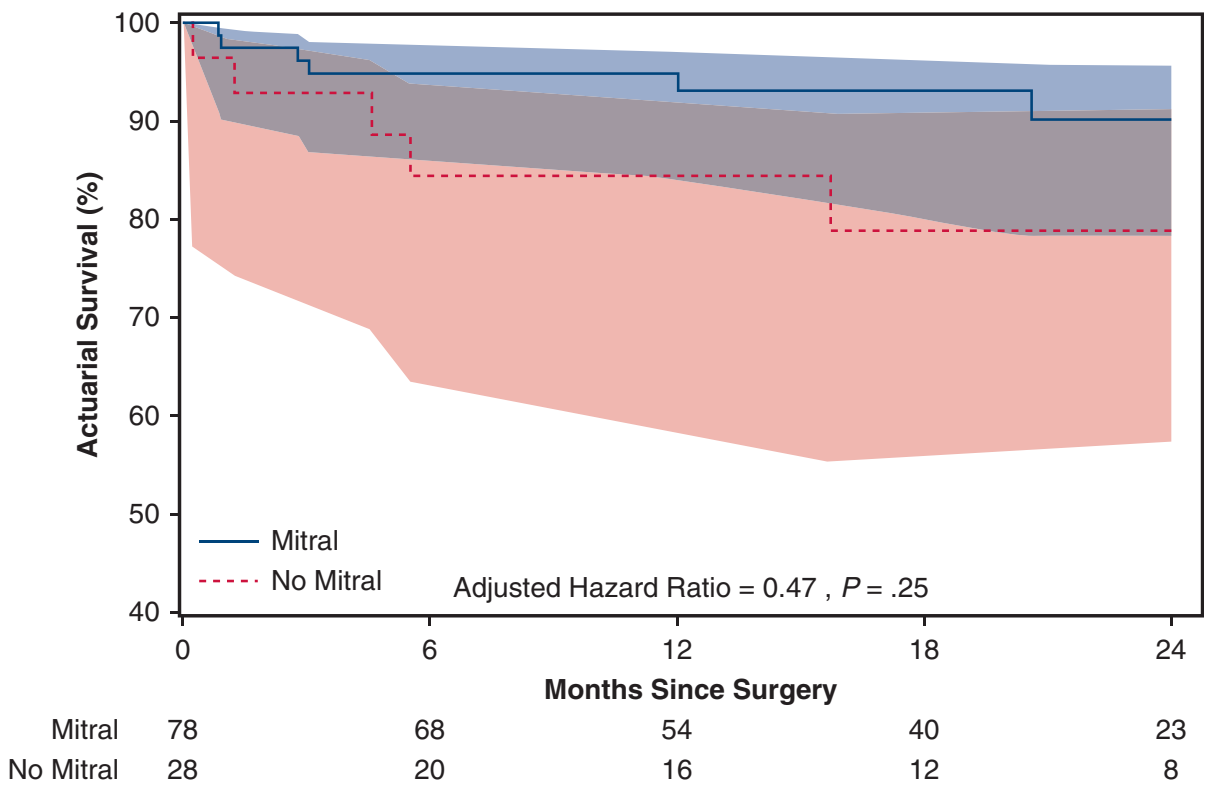

FIGURE 1. Survival estimate after left ventricular assist device implantation according to the concomitant mitral repair versus nonrepair. The shaded areas represent $95 \%$ confidence intervals. 


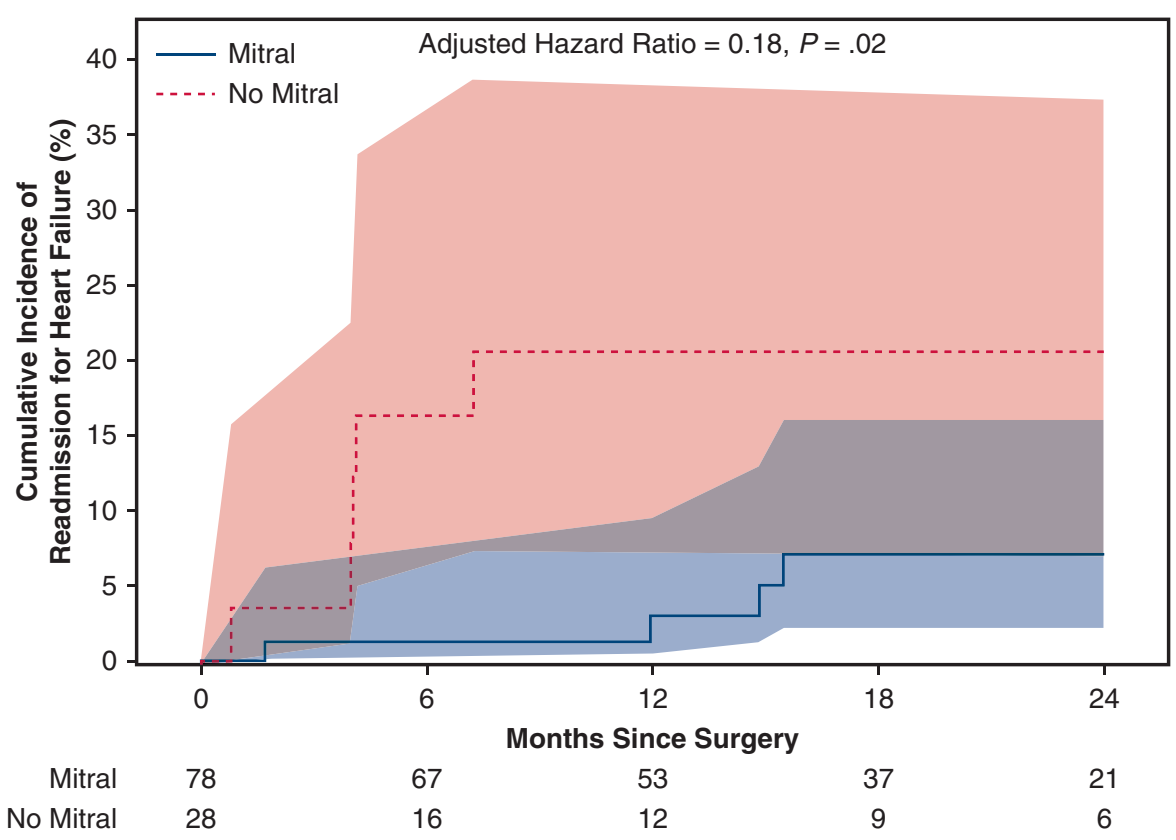

FIGURE 2. Cumulative incidence of readmission due to heart failure according to the concomitant mitral repair versus nonrepair. The shaded areas represent $95 \%$ confidence intervals.

suggest that there may be a subset in whom MR will not improve despite simple volume reduction of the left ventricle. These observations have led to the question as to whether more predictable elimination of MR (by valve repair or replacement at time of LVAD implantation) could result in improved clinical outcomes.

We have previously been liberal in the use of tricuspid valve repair to optimize right ventricular function after LVAD implantation. ${ }^{13}$ Encouraged by the work of Adamson and colleagues ${ }^{14}$ who reported use of MVr in $32 \%$ of LVAD recipients, we adopted routine $\mathrm{MVr}$, in addition to tricuspid valve repair, in patients with severe MR with the hope of reducing right ventricular afterload, promoting pulmonary vascular remodeling and optimizing right ventricular function. Our initial concern of adding a mitral procedure to an LVAD implantation was the potential for increase in complexity and risk of surgery. We were able to undertake these procedures with minimal incremental morbidity. Our mortality and morbidity was low, and in keeping with that of contemporary series of LVAD implantation. ${ }^{9,15}$

We believe that certain aspects of our approach are key to safely performing mitral surgery at time of LVAD implantation. Avoiding aortic clamping may help preserve right ventricular function by reducing intraoperative ischemic injury. With a trans-septal approach to the mitral valve, the valve can be exposed without excessive retraction and distortion of the aortic valve, allowing the procedure to be done without aortic clamping. We do not downsize the annuloplasty, so risk of inducing functional mitral stenosis should be minimal (we did not observe any cases of mitral stenosis in our series). Although downsizing is accepted as critical in valve repair for secondary mitral regurgitation, this should not apply to the LVAD setting, as the ventricle dimensions will decrease substantially with LVAD support, thus reducing the excessive tethering on the valve leaflets. Valve replacement could be a reasonable alternative to repair, but we believe repair is preferable to minimize valve-related complications, such as endocarditis, paravalvular regurgitation, and thromboembolism (which could negatively influence survival with an LVAD), so we reserve replacement for unrepairable valves. We believe exclusive use of transseptal and transapical approaches has contributed to our lack of bleeding complications directly related to the $\mathrm{MVr}$, because the only cardiac external suture lines remained the right atriotomy and the left ventriculotomy, which is no different than a standard LVAD implant with concurrent tricuspid valve repair. Furthermore, by avoiding an external suture line on the left atrium, doing a mitral repair should not complicate subsequent transplant because there is no suture material or breaching of tissue planes (and hence minimal scarring) in the interatrial groove.

Edge-to-edge repair may be a worthwhile alternative to ring annuloplasty in the LVAD setting, and seemed effective in our limited experience and is particularly attractive for sternal-sparing procedures. The edge-to-edge approach is quicker than annuloplasty and does not require separate septal or atrial incision. Edge-to-edge repair may not be applicable or effective in all cases, such as in the presence of thin leaflets, leaflet prolapse, or severe leaflet tethering. Further study is required to ascertain whether the edge-toedge and annuloplasty techniques are equivalent in safety and effectiveness. 


\section{Limitations}

This is a small, single-center retrospective observational series with inherent limitations. Our center is a mitral valve reference center with vast experience in primary $\mathrm{MVr}$, so we were able to seamlessly add a mitral annuloplasty to our LVAD implants. Our experience in non-LVAD MVr is a potential confounding factor and our results may not be generalizable to centers with less $\mathrm{MVr}$ experience. Our choice of devices and procedures was varied. However, although we used 3 devices, the predominant device used was HeartMate II $(90 \%)$. The study was performed over a 7 -year period and unmeasured confounders related to the accumulated experience, improvement in perioperative patient care, and changes in surgical techniques, may have influenced the outcomes. There was, for example, a lower threshold to do tricuspid valve repair in the mitral repair cohort. There were also some differences in the baseline status between the groups. However, we do not think that changes in management alone or differing patient cohort selection could explain the clinical, echocardiographic, and hemodynamic differences between our repair patients and controls, because we adjusted for these using multiple methods and on sensitivity analyses our observations remained robust. Our study has a relatively small sample size, and was not adequately powered to precisely quantify differences between the groups-larger studies will be required to elucidate more clearly the benefits of concurrent mitral valve repair.

\section{Future Directions}

There have been several prior small studies, although mostly uncontrolled, some suggesting a role for mitral surgery and others suggesting it is unnecessary. A recently published large multicenter analysis based on Interagency Registry for Mechanically Assisted Circulatory Support data included more than 250 mitral valve procedures and found lesser hospitalizations and improved functional status in patients who have $\mathrm{MVr},{ }^{16}$ thus supporting our findings. However, all these studies have been retrospectively analyzed. There remains an evidence gap, so there is clear need for prospective studies with randomized or nonrandomized controls. The Interagency Registry for Mechanically Assisted Circulatory Support database or Cardiothoracic Surgical Trials Network could form a repository for such a study where a subset of patients in designated centers with MR at LVAD implantation could be prospectively observed, systematically capturing hemodynamic, echocardiographic, clinical, and quality of life data. A sufficiently powered randomized trial, although ideal, is expensive and time-consuming and may not be a practical priority in the LVAD community at this time.

\section{CONCLUSIONS}

Concurrent MVr at the time of LVAD implantation can be done without substantial increase in perioperative adverse events. Our data suggest MVr could have potential benefit in terms of greater reversal of pulmonary hypertension and reduction in hospitalizations for heart failure. Surgical treatment of MR at the time of LVAD may be worth particular consideration in patients with severe MR who are expected to be supported by the LVAD for a prolonged time period.

\section{Webcast}

You can watch a Webcast of this AATS meeting presentation by going to: https://aats.blob.core.windows.net/ media/18May01/23BC\%203.MCS\%20Transplant/S96_6_ webcast_052307017.mp4.

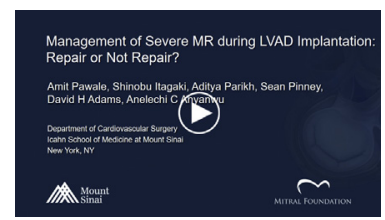

\section{Conflict of Interest Statement}

The Icahn School of Medicine at Mount Sinai receives royalty payments from Edwards Lifesciences and Medtronic for intellectual property related to Dr Adams' involvement in the development of 2 mitral valve repair rings and 1 tricuspid valve repair ring. All other authors have nothing to disclose with regard to commercial support

The authors thank Nana Toyoda, MD, PhD, for providing statistical analysis.

\section{References}

1. Firstenberg MS, Sai-Sudhakar CB, Anyanwu A. Valvular disease in patients requiring long-term left ventricular assist devices: pathophysiology and therapeutic options. Expert Rev Cardiovasc Ther. 2012;10:205-13.

2. Pal JD, Klodell CT, John R, Pagani FD, Rogers JG, Farrar DJ, et al. Low operative mortality with implantation of a continuous-flow left ventricular assist device and impact of concurrent cardiac procedures. Circulation. 2009;120:215-9.

3. Ammirati E, Musca F, Cannata A, Garascia A, Verde A, Pacher V, et al. Limited changes in severe functional mitral regurgitation and pulmonary hypertension after left ventricular assist device implantation: a clue to consider concurrent mitral correction? Int J Cardiol. 2013;167:e35-7.

4. Taghavi S, Hamad E, Wilson L, Clark R, Jayarajan SN, Uriel N, et al. Mitral valve repair at the time of continuous-flow left ventricular assist device implantation confers meaningful decrement in pulmonary vascular resistance. ASAIO J. 2013;59:469-73

5. Zoghbi WA, Enriquez-Sarano M, Foster E, Grayburn PA, Kraft CD, Levine RA, et al. Recommendations for evaluation of the severity of native valvular regurgitation with two-dimensional and Doppler echocardiography. J Am Soc Echocardiogr. 2003; 16:777-802.

6. Pawale A, Plotkina I, Anyanwu AC. Technique for implantation of HeartMate II left ventricular assist device with concurrent mitral and tricuspid valve repair. Ann Cardiothorac Surg. 2014;3:532-4.

7. Fine JP, Gray RJ. A proportional hazards model for the subdistribution of a competing risk. J Am Stat Assoc. 1999;94:496-509.

8. Elze MC, Gregson J, Baber U, Williamson E, Sartori S, Mehran R, et al. Comparison of propensity score methods and covariate adjustment: evaluation in 4 cardiovascular studies. J Am Coll Cardiol. 2017;69:345-57.

9. Stulak JM, Tchantchaleishvili V, Haglund NA, Davis ME, Schirger JA, Cowger JA, et al. Uncorrected pre-operative mitral valve regurgitation is not 
associated with adverse outcomes after continuous-flow left ventricular assist de vice implantation. J Heart Lung Transplant. 2015;34:718-23.

10. Jelenc M, Jelenc B, Vrtovec B, Kneževič I. Mitral regurgitation and axial flow left ventricular assist device: a computer simulation study. ASAIO J. 2013;59: 405-9.

11. Kemal HS, Ertugay S, Kahraman U, Engin C, Nalbantgil S, Yagdi T, et al. Impact of residual mitral regurgitation on right ventricular systolic function after left ventricular assist device implantation. J Heart Lung Transplant. 2015;34:S167.

12. Kitada S, Kato TS, Thomas SS, Conwell SD, Russo C, Di Tullio MR, et al. Preoperative echocardiographic features associated with persistent mitral regurgitation after left ventricular assist device implantation. J Heart Lung Transplant. 2013;32:897-904.

13. Krishan K, Nair A, Pinney S, Adams DH, Anyanwu AC. Liberal use of tricuspidvalve annuloplasty during left-ventricular assist device implantation. Eur J Cardiothorac Surg. 2012:41:213-7.

14. Adamson RM, Stahovich M, Chillcott S, Baradarian S, Chammas J, Jaski B, et al Clinical strategies and outcomes in advanced heart failure patients older than 70 years of age receiving the HeartMate II left ventricular assist device: a community hospital experience. J Am Coll Cardiol. 2011:57:2487-95.

15. Cowger J, Sundareswaran K, Rogers JG, Park SJ, Pagani FD, Bhat G, et al. Predicting survival in patients receiving continuous flow left ventricular assist devices: the HeartMate II risk score. J Am Coll Cardiol. 2013;61:313-21.

16. Robertson JO, Naftel DC, Myers SL, Tedford RJ, Joseph SM, Kirklin JK, et al Concomitant mitral valve procedures in patients undergoing implantation of continuous-flow left ventricular assist devices: an INTERMACS database analysis. J Heart Lung Transplant. 2018;37:79-88.

Key Words: left ventricular assist device, mitral regurgitation, mitral valve repair

\section{Discussion}

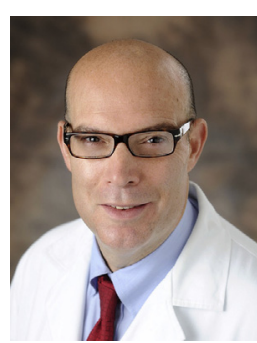

Dr Scott Silvestry (Orlando, Fla). Thank you for the opportunity to discuss this paper and thank you as well for the copy of the manuscript ahead of time. In our paper that we published in the Journal of Heart and Lung Transplantation in January, we found nearly the same results in that no survival difference and a suggestion of delay to readmission in a large trial, a large database.

In smaller studies there are often large amounts of complementary data. There is a lot of granularity that singleinstitution studies can add that multicenter studies or large registries cannot. Do you have any data regarding length of stay, intensive care unit (ICU) hours, and resource utilization in these patients for the mitral group versus the nonmitral group?

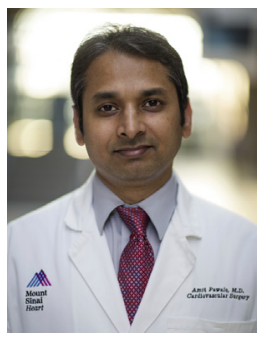

Dr Pawale (New York, NY). The Interagency Registry for Mechanically Assisted Circulatory Support database is a multicenter registry with a vast array of surgeons and surgical techniques, inclusion criteria, and postoperative management. We believe that a small, single-center study can still be useful in assisting the early efficacy of a technique postoperatively because of the homogenous approach of the management.

So in terms of ICU stay, the median length of ICU stay for the mitral valve repair group was 4 days and the no mitral valve repair group was 6 days. The median hospital stay for the mitral valve repair group was 16 days and 24 days for the no mitral valve repair group. Incidence of renal replacement therapy was $5 \%$ in the mitral group compared with $14 \%$ in the nonmitral group.

Dr Silvestry. Thank you. Those are very interesting numbers. Lastly, do you believe that your control group/ comparative group of patients was appropriate given that the central venous pressure was higher and there were other significant differences between the groups?

Dr Pawale. The fact that the control study is historical and the patients sicker is the major limitation of our study. 
TABLE E1. Echocardiographic parameters at 3, 6, and 12 months of follow-up according to the concomitant mitral repair versus nonrepair

\begin{tabular}{|c|c|c|c|c|c|c|c|c|}
\hline Repair & $\begin{array}{c}\text { MR } \\
\text { (more than mild) }\end{array}$ & $P$ value & $\begin{array}{c}\text { TR } \\
\text { (more than mild) }\end{array}$ & $P$ value & $\begin{array}{c}\text { RV dysfunction } \\
\text { (moderate or greater) }\end{array}$ & $P$ value & LVEDD (cm) & $P$ value \\
\hline 3 mo (median, $3.8 \mathrm{mo}$ ) & & .001 & & .72 & & .05 & & .82 \\
\hline Mitral & 0/53 (0) & & 8/51 (16) & & $40 / 51(78)$ & & 5.8 & \\
\hline Nonmitral & $7 / 24(29)$ & & $3 / 24(13)$ & & $13 / 23(57)$ & & 5.8 & \\
\hline 6 mo (median, $6.1 \mathrm{mo}$ ) & & .12 & & 1.00 & & .40 & & .05 \\
\hline Mitral & $0 / 14(0)$ & & $1 / 10(10)$ & & $8 / 10(80)$ & & 5.7 & \\
\hline Nonmitral & $4 / 10(40)$ & & 2/14 (14) & & $9 / 14(64)$ & & 6.6 & \\
\hline 12 mo (median, $14.2 \mathrm{mo})$ & & .006 & & 1.00 & & .08 & & .83 \\
\hline Mitral & $0 / 35(0)$ & & $7 / 35(20)$ & & 28/35 (80) & & 5.9 & \\
\hline Nonmitral & 3/9 (33) & & $2 / 9(22)$ & & $4 / 8(50)$ & & 5.8 & \\
\hline
\end{tabular}

Values are presented as $\mathrm{n} / \mathrm{n}(\%) . M R$, Mitral regurgitation; $T R$, tricuspid regurgitation; $R V$, right ventricle; $L V E D D$, left ventricular end-diastolic diameter.

TABLE E2. Adjusted hazard ratio (aHR) of readmission from each sensitivity analyses

\begin{tabular}{|c|c|c|}
\hline Method & aHR $(95 \%$ confidence interval $)$ & $P$ value \\
\hline Multivariable subdistribution hazard model with limited number of variables & $0.21(0.05-0.86)$ & .031 \\
\hline Propensity score adjusted model & $0.18(0.06-0.60)$ & .0051 \\
\hline Propensity score adjusted model with doubly robust approach* & $0.17(0.04-0.75)$ & .019 \\
\hline
\end{tabular}

